\title{
GMR
}

\section{Digital expression analysis of the genes associated with salinity resistance after overexpression of a stress-responsive small GTP-binding RabG protein in peanut}

\author{
J.M. Sui ${ }^{1 *}$, G. Li ${ }^{1 *}$, G.X. Chen ${ }^{1}$, M.Y. Yu ${ }^{1}$, S.T. Ding ${ }^{2}$, J.S. Wang ${ }^{1}$ and \\ L.X. Qiao ${ }^{1}$ \\ ${ }^{1}$ College of Life Science, Qingdao Agricultural University, \\ Key Laboratory of Qingdao Major Crop Germplasm Resource Innovation and \\ Application, Key Lab of Plant Biotechnology in Universities of Shandong, \\ Qingdao, Shandong, China \\ ${ }^{2}$ College of Foreign Languages, Qingdao Agricultural University, \\ Qingdao, Shandong, China
}

*These authors contributed equally to this study.

Corresponding author: L.X. Qiao

E-mail:1xqiao73@163.com

Genet. Mol. Res. 16 (1): gmr16019432

Received October 13, 2016

Accepted December 14, 2016

Published March 8, 2017

DOI http://dx.doi.org/10.4238/gmr16019432

Copyright (C) 2017 The Authors. This is an open-access article distributed under the terms of the Creative Commons Attribution ShareAlike (CC BY-SA) 4.0 License.

\begin{abstract}
The Rab protein family is the largest family of the small GTP-binding proteins. Among them, the RabG genes are known to be responsive to abiotic stresses, but the molecular mechanisms of the stress responses mediated by RabG genes in plants is poorly understood. To investigate the molecular mechanism of $A h R a b G$ gene in peanut, transgenic plants overexpressing the $A h R a b G$ gene (S6) with relatively higher salinity resistance than the non-transgenic plants (S7) were obtained. Digital gene expression (DGE) sequencing was performed with the leaves of S6 and S7 plants before and after
\end{abstract}

Genetics and Molecular Research 16 (1): gmr16019432 
salinity-stress treatment. The $A h R a b G$ gene in peanut was found to be involved in a few pathways such as "photosynthesis", "oxidative phosphorylation", "AMPK signaling pathway", "plant hormone signal transduction", etc. A total of 298 differentially expressed genes (DEGs) were found to be upregulated or downregulated at five sampling time points based on the comparison between S6 and S7 plants. Among them, 132 DEGs were responsive to salinity stress in S6 and/or S7 after salinity-stress treatment. These 132 DEGs included genes encoding various transcription factors and proteins involved in resistance to salinity stress such as MYB, AP2, RING-H2 zinc finger proteins, late embryogenesis abundant (LEA) proteins, dehydration-responsive protein RD22, peroxidases, CBL-interacting protein kinases, calciumbinding proteins, and others. The information from this study will be useful for further studies on elucidating the mechanism of salinity resistance conferred by $R a b G$ genein peanut.

Key words: Peanut (Arachis hypogaea L.); RabG; Overexpression; Salinity stress; Digital gene expression; Differentially expressed genes

\section{INTRODUCTION}

The small GTP-binding proteins with a molecular mass ranging from 20 to $30 \mathrm{kDa}$ are ubiquitous among eukaryotes (Lowy and Willumsen, 1993). They can be divided into Ras, Rho/Rac, Ypt/Rab, Ran/TC4, and Arf/Sar subfamilies, and each of them plays a distinct role in various cellular functions. Among these, the Rab proteins constitute the largest subfamily of the small GTP-binding proteins (Brighouse et al., 2010).

The Rab proteins participate in the vesicle formation, transportation, and docking during exocytic and endocytic cycles (Pereira-Leal and Seabra, 2001). Furthermore, they are involved in signal transduction, cytoskeletal organization (Nuoffer and Balch, 1994), pathogen response (Sano et al., 1994; Zhao et al., 2015), and responses to various environmental stimuli (Xing et al., 1997; Nicolás et al., 1998; O’Mahony and Oliver, 1999; Bolte et al., 2000). Among different Rab proteins, the Rab7 (G)-related proteins regulate the delivery of the internalized materials into the degradative compartments and the acquisition of lysosomal hydrolases (Bogdanovic et al., 2000). In yeast, a homologue of Rab7, namely Ypt7p, localizes to vacuoles and regulates the homotypic fusion between vacuolar compartments (Schimmöller and Riezman, 1993). The Ypt7 mutant is characterized by highly fragmented vacuoles and differential defects in vacuolar protein transport and maturation (Wichmann et al., 1992).

Interestingly, some of the Rab7 genes are found to be responsive to various environmental stimuli including drought, salt, extreme temperature, abscisic acid (ABA) application, and elicitors (Nahm et al., 2003; Mazel et al., 2004; Agarwal et al., 2008; Rajan et al., 2015). For example, the rice Rab7 homolog, OsRab7 transcript, was strongly induced by dehydration and ABA treatment, but not by salt treatment. Transgenic plants overexpressing the OsRab7 gene showed enhanced growth and increased proline content at the seedling stage after salt stress treatment. Moreover, an increase in the number of vesicles was observed in the root tip of transgenic rice overexpressing the OsRab7 gene (Nahm et al., 2003). The AtRabG3e gene from Arabidopsis was induced during the programmed cell death following

Genetics and Molecular Research 16 (1): gmr16019432 
treatment with superoxide or salicylic acid, or infection with necrogenic pathogens. The transgenic plants expressing the AtRabG3e gene exhibited accelerated endocytosis, increased tolerance to salt and osmotic stresses, and reduced accumulation of reactive oxygen species under salt-treated conditions (Mazel et al., 2004). The expression of PgRab7 transcript from Pennisetum glaucum increased drastically by various environmental stimuli such as salt, cold, dehydration, and a plant hormone indole-3-acetic acid (IAA). Transgenic tobacco overexpressing $P g R a b 7$ gene showed enhanced tolerance to $\mathrm{NaCl}$ and mannitol as well as increased alkaline phosphatase activity (Agarwal et al., 2008). The transcript expression of the AlRab7 gene from Aeluropus lagopoideswas differentially regulated by dehydration, salinity, and ABA. The recombinant Escherichia coli harboring the AlRab7 gene showed improved growth in the medium containing salt, mannitol, ABA, and IAA (Rajan et al., 2015).

In our previous study, we isolated an important small GTP-binding protein from peanut; this protein was found to be responsive to different stress treatments and plant hormone ABA, and exhibited enhanced tolerance to different stresses in E. coli and transgenic peanut (Song et al., 2012). To elucidate the mechanism of $A h R a b G$ in peanut, we prepared transgenic plants overexpressing the $A h R a b G$ gene and non-transgenic plants (as control), and determined the dynamic differences in their digital gene expression (DGE) profiles before and after salinity stress treatment. The specific pathways regulated by $A h R a b G$ were identified. The possible roles of the differentially expressed genes (DEGs) were discussed.

\section{MATERIAL AND METHODS}

\section{Plant growth and stress treatments}

Transgenic peanut plants overexpressing the $A h R a b G$ gene (S6, the level of $A h R a b G$ gene was 1.5 times that of control) and peanut cultivars 'Xuzhou68-4' used as control plants (S7) were obtained from Qingdao Agricultural University, China. The seeds of each genotype were grown in a growth chamber with dark/light cycle of $8 / 16 \mathrm{~h}$ at a temperature of $28^{\circ} \mathrm{C}$ for six weeks. Subsequently, the seedlings of the S6 and S7 plants were irrigated with $250 \mathrm{mM}$ $\mathrm{NaCl}$ for salinity stress under the same conditions. After exposure to a salinity stress for 0 , $6,12,24$, and $48 \mathrm{~h}$, the leaves of the S6 and S7 seedlings were removed and placed in liquid nitrogen until further use.

\section{Library construction for transcriptome sequencing and functional annotation}

Total RNA was isolated from the sampled leaves with Trizol reagent (Invitrogen, Carlsbad, CA, USA). The RNA from each combination of seedling type (S6 or S7) after salt treatment was pooled, and then analyzed with an Agilent 2100 Bioanalyzer (Agilent Technologies, Santa Clara, CA, USA). RNA sequencing was carried out by Novogene Bioinformatics Technology Co., Ltd. (Beijing, China) on an Illumina HiSeq2000 sequencer. The 125-bp paired-end reads were generated. All clean sequence read data were deposited in the sequence read archive (SRA) database (accession number SRR3114511) of the National Centre for Biotechnology Information (NCBI). Subsequently, they were assembled into comprehensive unigenes by means of the Trinity and TGICL programs. The functional annotation of these genes was conducted with reference to various online databases such as NR, NT, SwissProt, PFAM, KOG, GO, and KEGG (Sui et al., unpublished data).

Genetics and Molecular Research 16 (1): gmr16019432 


\section{DGE sequencing and mapping}

The RNA samples from the S6 and S7 genotypes were labeled with the sampling times $(0,6,12,24$, and $48 \mathrm{~h}$ after $\mathrm{NaCl}$ treatment) as follows: S6_0, S6_6, S6_12, S6_24, and S6_48 for the S6 genotype, and S7_0, S7_6, S7_12, S7_24, and S7_48 for the S7 genotype. Each combination of genotype and sampling time after salt treatment was represented by two replicate RNA samples. DGE sequencing was carried out by Novogene Institute (Beijing, China) on an Illumina HiSeq2000 sequencer with a single 50-bp end read for each reaction. All reads of each library were deposited in the NCBI SRA database (accession number SRR3210688 and SRR3204350) and mapped onto the unigenes. The unigene expression was normalized with the value of RPKM (reads per kilobase per million mapped reads).

\section{Identification of differentially expressed genes (DEGs)}

Differential expression analysis was compared both within each genotype and between the two genotypes using the DESeq R package (1.10.1). The DESeq package provides statistical routines for determining differential expression in the digital gene expression data using a model based on the negative binomial distribution (Anders and Huber, 2010). The resulting $\mathrm{P}$ values were adjusted using the Benjamini-Hochberg approach for controlling the false discovery rate (Benjamini and Hochberg,1995). Between the genotypes S6 and S7, the gene expression was compared at $0,6,12,24$, and $48 \mathrm{~h}$. If the level of expression was significantly different (the adjusted $\mathrm{P}$ value $<0.05$ ) in a comparison, then the gene was considered to be differentially expressed. Within the genotypes S6 and S7, the gene expression was compared between two sampling times. If the level of expression was significantly up- or downregulated (the adjusted $\mathrm{P}$ value $<0.05$ ) in a comparison, then the gene was considered to be responsive to salinity stress. Pathways that were statistically significant (false discovery rate (FDR) $\leq 0.05$ ) were enriched with KEGG pathways.

\section{RT-qPCR analysis}

Reverse transcription was performed using the SuperScript Reverse Transcriptase Kit (Invitrogen). The primers were designed using the Oligo6 software. For RT-qPCR, the SYBR ${ }^{\circledR}$ Premix Ex Taq ${ }^{\mathrm{TM}}$ (TAKARA) was used on a Bio-Rad CFX96 real-time PCR detection system (Bio-Rad, Hercules, CA, USA). Gene expression was analyzed for S6 and S7 samples at 6, 12, 24 , and $48 \mathrm{~h}$ after the application of the salinity-stress treatment. All reactions for each gene were performed in three biological replicates with a $20-\mu \mathrm{L}$ reaction volume. The parameters of the thermal cycle were: initial denaturation at $95^{\circ} \mathrm{C}$ for $2 \mathrm{~min}$, followed by 40 cycles of denaturation at $95^{\circ} \mathrm{C}$ for $40 \mathrm{~s}$ and annealing at $50^{\circ}-56^{\circ} \mathrm{C}$ for $40 \mathrm{~s}$. The relative expression levels were calculated using the $2^{\text {r } \triangle \mathrm{Ct}}$ method, with normalization to the internal reference $\beta$-actin gene from peanut as described previously (Livak and Schmittgen, 2001).

\section{RESULTS}

To investigate the gene expression patterns of each genotype before and after the salinity-stress treatment, 20 DGE libraries were constructed and sequenced with the method of Illumina deep sequencing technology. For each sample, it generated 11,037,884-13,409,746

Genetics and Molecular Research 16 (1): gmr16019432 
clean reads and 0.55-0.67 $\mathrm{G}$ clean bases. In the S6 genotype, the number of unigenes (FPKM $>0.3$ ) ranged from 49,755 to 53,353 with an average value of 51,371, whereas in the S7 genotype, the number of unigenes (FPKM $>0.3$ ) ranged from 49,695 to 54,337 with an average value of 51,479 (Table S1).

\section{Clustering analysis of samples and identification of DEGs}

To obtain a global view of the gene expression profiles of the S6 and S7 genotypes, we analyzed the gene expression profiles for 10 samples from both genotypes using the method of clustering algorithms and treeview. Generally, similar gene expression patterns were found between S6 and S7 before and after salinity stress at paired time points. The DEG profiles at the 6 and $12 \mathrm{~h}$ were significantly different from those before the stress treatment. With an increase in the treatment time, the tendency of DEGs became similar (Figure 1).

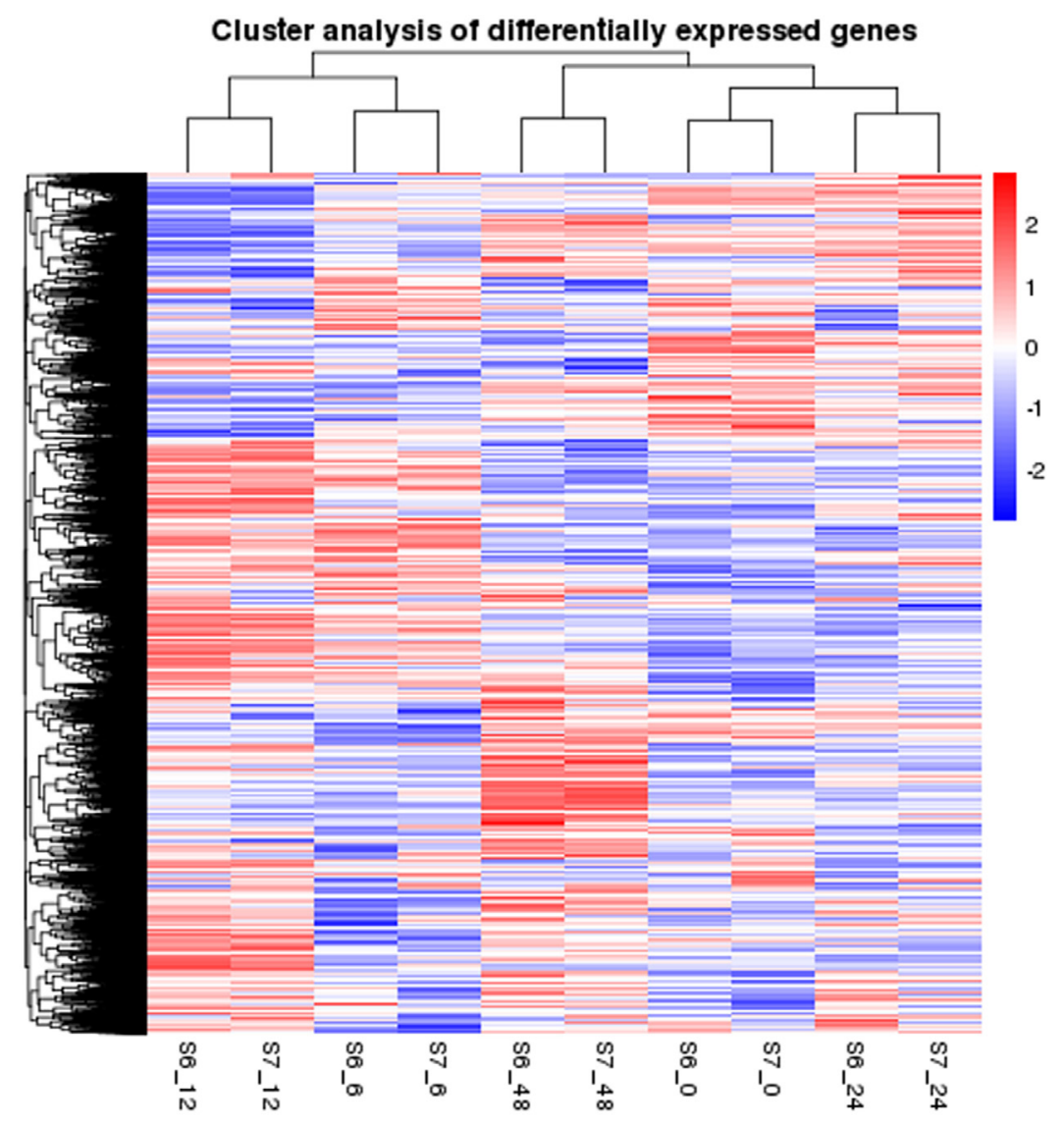

Figure 1. Hierarchical clustering analysis of salinity-induced changes in gene expression in peanut leaves. S6, transgenic peanut plants overexpressing $A h R a b G$ gene; S7, Xuzhou68-4 (control); the first letter plus number refers to the S6 or S7 sample and the second number refers to the time (from 0 to $48 \mathrm{~h}$ ) after salinity stress treatment. Each value of gene expression is an average of the two replications. Red bar indicates upregulated expression, and blue bar indicates downregulated expression. 
In the present study, the DEGs were identified with the criteria of the adjusted $\mathrm{P}$ value less than 0.05 . The differential comparisons between the control and salinity stress-treated samples were shown in the S6 and S7 series data sets. These 10 series data sets represented the DEGs of both genotypes before and after salinity stress treatment.

The dynamic trends of DEGs in the S6 and S7 data sets were investigated. Compared with control at $0 \mathrm{~h}$, the differentially expressed genes showed peaks after treatment with salinity stress, and the number of downregulated DEGs was markedly higher than the upregulated ones at 12 and $48 \mathrm{~h}$ in S6 and S7 genotypes. The total DEGs, downregulated as well as upregulated, showed similar changing trends at $0,6,12,24$ and $48 \mathrm{~h}$ in S6 and S7 genotypes (Figure 2). A total of 217 DEGs in the S6 genotype (Figure 3A) and 655 DEGs in the S7 genotype (Figure 3B) were found to be common at four different time points after salinity stress treatment.

\begin{tabular}{|c|c|c|c|c|c|c|c|c|c|c|}
\hline & \multicolumn{5}{|c|}{ DEGs in 56} & \multicolumn{5}{|c|}{ DAGs in 57} \\
\hline Tine (h) & O & 6 & 12 & 24 & 48 & $\mathbf{0}$ & 6 & 12 & 24 & 48 \\
\hline $\mathbf{0}$ & 0 & 1581 & 1859 & 516 & 869 & $\mathbf{0}$ & 2321 & 2889 & 1402 & 1683 \\
\hline 6 & 1624 & $\mathbf{0}$ & 231 & 748 & 751 & 2443 & $\mathbf{0}$ & 838 & 1682 & 1650 \\
\hline 12 & 2667 & 680 & 0 & 2012 & 1056 & 3190 & 1166 & $\mathbf{0}$ & 2661 & 1958 \\
\hline 24 & 407 & 775 & 1340 & O & 323 & 1420 & 1797 & 2190 & $\mathbf{0}$ & 1111 \\
\hline 48 & 1113 & 880 & 859 & 380 & 0 & 1904 & 1794 & 1874 & 1143 & 0 \\
\hline
\end{tabular}

Figure 2. Changes in the numbers of differentially expressed gene (DEGs) in the S6 and S7 genotypes of peanut following salinity-stress treatment. S6 overexpresses the $A h R a b G$ gene, while S7 does not. Orange indicates upregulated expression, and blue indicates downregulated expression.

DEGs were also identified by comparison of gene expression between S6 and S7 at each sampling time. The data set at $6 \mathrm{~h}$ showed the least exclusive DEGs, and they were mostly exclusive in the data sets at 0 and $24 \mathrm{~h}$. Interestingly, three DEGs were found to be common in all five data sets (Figure 3C).

\section{Annotation and KEGG enrichment analyses of DEGs based on S6 vs. S7 comparison}

For functional annotation of the DEGs that were determined by comparison of gene expression in S6 vs. S7, the KEGG enrichment analyses were mainly referenced. At $0 \mathrm{~h}$ (before salinity stress treatment), 13 downregulated and 12 upregulated DEGs were screened. The main pathways included "photosynthesis", "oxidative phosphorylation", "AMPK signaling pathway", and "plant hormone signal transduction" (Figure 4A). Interestingly, the enrichment analyses indicated that all differentially regulated DEGs involved in "photosynthesis" and "oxidative phosphorylation" pathways were upregulated after overexpression of the RabG genes. After $6 \mathrm{~h}$ of salt stress, five downregulated and two upregulated DEGs were assigned the KEGG pathways. Among the five downregulated DEGs, three DEGs were involved in the "circadian rhythm" pathway and two DEGs (c39883_g1 and c35382_g1) were involved in the "flavonoid biosynthesis" pathway. One DEG (c34015_g1) was found to be involved in three pathways, including "GABAergic synapse", "synaptic vesicle cycle", and "retrograde endocannabinoid signaling" (Figure 4B). After $12 \mathrm{~h}$ of salt stress, five Tand Gs ously. bacamef treatment time, downregulated DEGs were assigned the KEGG pathways (Figure 4C). Among them, two DEGs, which were also found to be downregulated at $6 \mathrm{~h}$, were involved in the "circadian rhythm" and "flavonoid biosynthesis" pathways. A DEG (c19905_g1), which encoded homogentisate phytyltransferase, was also found to be downregulated at 0 and $6 \mathrm{~h}$. Another DGE (c32262_g1), which was involved in the "thiamine metabolism" and "terpenoid

Genetics and Molecular Research 16 (1): gmr16019432 


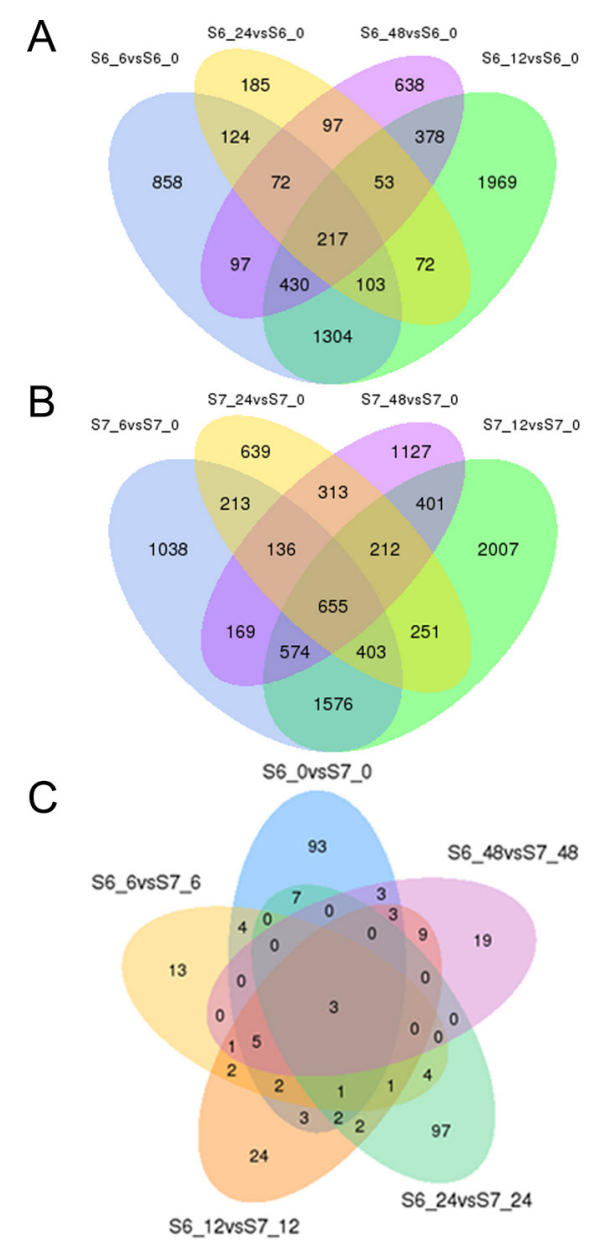

Figure 3. Venn diagrams indicating the numbers of differentially expressed genes (DEGs) in: A. the S6 genotype (after $v s$. before the salinity-stress treatment; B. the S7 genotype (after $v s$. before the salinity-stress treatment); and C. S6 vs. S7 (after the salinity-stress treatment). The numbers of DEGs exclusively expressed in one comparison are shown in the non-overlapping regions. The numbers of DEGs with a common tendency of expression change in the comparisons are shown in the overlapping regions.

backbone biosynthesis" pathways, was also found to be downregulated at 6 and $12 \mathrm{~h}$. After $24 \mathrm{~h}$ of salt stress, four upregulated and 22 downregulated DEGs were assigned the KEGG pathways, and they were mainly involved in the "plant-pathogen interaction," "PI3K-Akt signaling pathway", "protein processing in endoplasmic reticulum", "plant hormone signal transduction", "thyroid hormone synthesis", "circadian rhythm-plant", "antigen processing and presentation", "signaling pathways regulating pluripotency of stem cells", etc. (Figure 4D). One DGE (C34875_g1), which encoded the vacuolar-processing enzyme (dynein heavy chain of dynein motor), was involved in the "antigen processing and presentation" and "lysosome" pathways. After $48 \mathrm{~h}$ of salt stress, 43 DEGs were screened, but none of them were found in the KEGG enrichment analysis.

Genetics and Molecular Research 16 (1): gmr16019432 
A

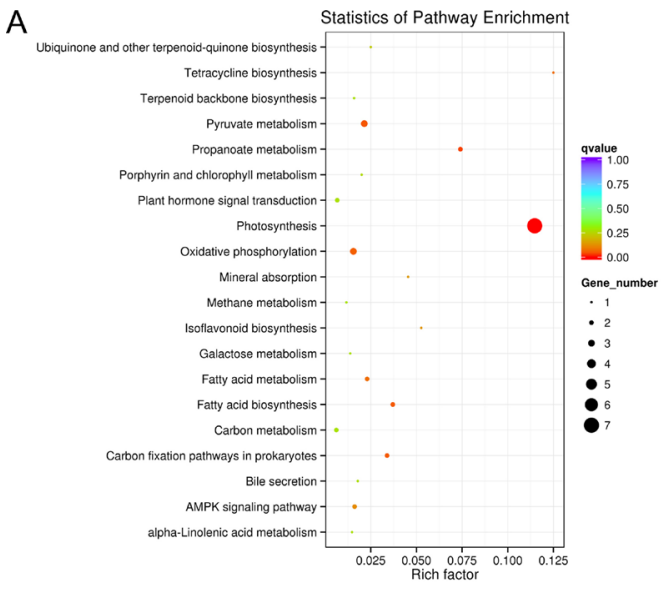

C

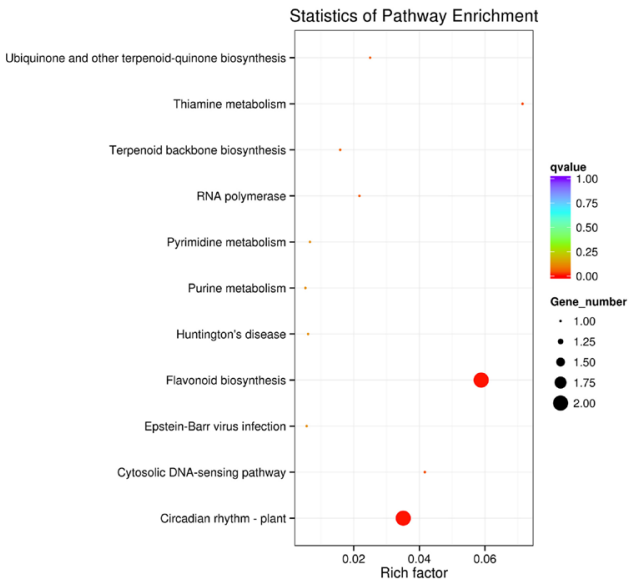

B

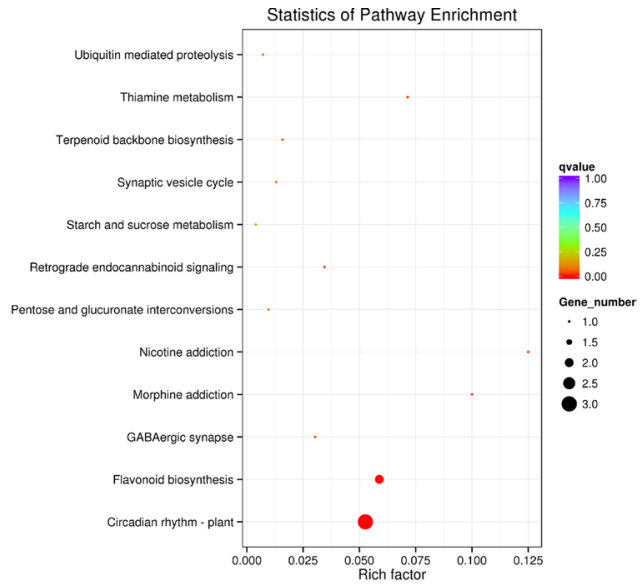

D

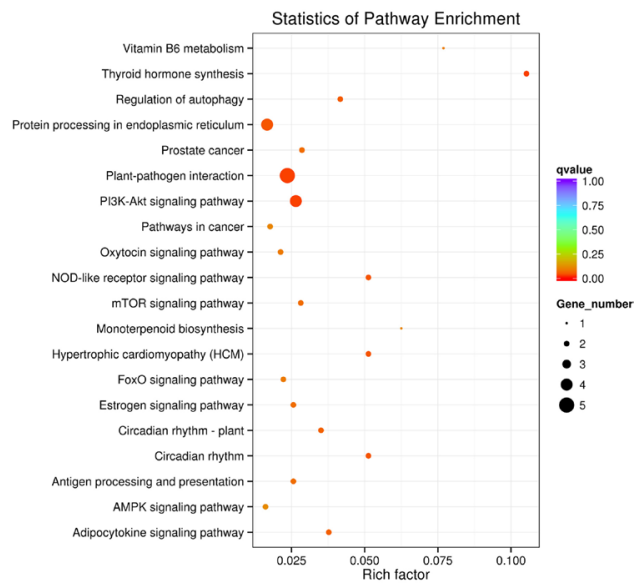

Figure 4. KEGG Pathway enrichment analyses of differentially expressed genes (DEGs) in D_6 (A), D_12 (B), D_24 (C), and D_48 (D) based on the comparison of S6 vs S7 genotypes of peanut.

\section{Screening of salinity-responsive DEGs based on S6 vs. S7 comparison}

To further elucidate the salinity-resistant mechanism of the $R a b G$ gene in peanut, the salinity-responsive DEGs were screened on the basis of S6 vs S7 comparison. Of all the 298 DEGs at five sampling times (Table S2 and Figure 3), 132 DEGs, whose expression were significantly up- or downregulated in S6 relative to S7, were responsive to the salinity stress at $6,12,24$, and $48 \mathrm{~h}$ (Table S3 ). The expression patterns of these genes taking key roles in plant resistance to salinity stress were investigated. Among them, 66 DEGs (9 in the S6 genotype and 57 in the S7 genotype) were sensitive to the salinity stress treatment, and 15 among them were sensitive at two sampling times.

\section{Experimental verification of DEGs}

To determine whether our RNA-seq identification in peanut was reliable, we used

Genetics and Molecular Research 16 (1): gmr16019432 
RT-qPCR to monitor the expression pattern of eight DEGs according to the result of S6 vs S7 comparison at $0,6,12,24$, and $48 \mathrm{~h}$ sampling times; the expression of these genes was quantified in both S6 and S7 genotypes. The primers of the selected genes are listed in Table S4. Their expression levels, as determined by RT-qPCR, were correlated with the results of the RNA-seq (Table S5).

\section{DISCUSSION}

Our DGE sequencing identified some previously reported stress-related transcription factors (TFs) and genes after overexpressing the $A h R a b G$ gene from peanut. Five TFs, including the ethylene-responsive transcription factor AP2, MYB, and RING-H2 zinc finger protein, were differentially expressed under salinity stress between S6 and S7 genotypes. The MYB TFs have been found to play key roles in both the repression and derepression of the responses to salt stress as well as other abiotic stresses (Lu et al., 2014). An R2R3type MYB TF from rice, OsMPS, which might simultaneously play roles in multiple stressinduced signaling pathways, was found to regulate rice tolerance to salinity, plant hormones, and cell wall synthesis (Schmidt et al., 2013). We found thatone MYB TF (c38087_g1) was upregulated at $6 \mathrm{~h}$, while the other MYB TF (c41425_g1) was downregulated at $24 \mathrm{~h}$. One AP2 TF (32271_g1) was found to be upregulated at $6 \mathrm{~h}$ [the log2 (fold-change) >3]. The AP2 TFs have been proved to be involved as cross-talk factors in various biotic and abiotic stresses and plant hormone signal transduction (Fujita et al., 2006). For example, an ethylene response factor (ERF)-typeAP2 TF from soybean (GmERF3) was induced by various kinds of stress treatments mentioned above (Zhang et al., 2009). Two DEGs encoding RING-H2 zinc finger proteins (c37125_g2 and c35368_g1) were differentially expressed between the S6 and S7 genotypes after salinity stress.

Some genes that were differentially expressed in S6 vs S7 comparison were screened to be responsive to the salinity stress. These included DEGs encoding LEA proteins, dehydration-responsive protein RD22, and peroxidase. Among these genes, three $L E A$ genes (c32599_g1, c30685_g1, and c35561_g1) were significantly upregulated in S6 relative to S7 [the $\log 2$ (fold-change) $>2$ ]. In plants, the LEA proteins can accumulate to high levels during the last stage of seed maturation and under abiotic stresses, playing a protective role during the damage caused by environmental stresses (Battaglia et al., 2008). The gene encoding RD22 (c65965_g1) was downregulated in S6 relative to the S7 genotype at $12 \mathrm{~h}$. Its expression level was extremely downregulated after the salinity-stress treatment as compared to its expression before treatment in S6 and S7, especially at $48 \mathrm{~h}$, and this was in agreement with the findings of HbRD22 gene from Hevea brasiliensis exposed to drought stress (Gao et al., 2010), but the opposite result was obtained in the other species such as Arabidopsis. The result showed that the RD22 gene (c65965_g1) from peanut might be a negative regulator under salinity stress. Peroxidases are considered to be bifunctional enzymes that not only oxidize various substrates in the presence of $\mathrm{H}_{2} \mathrm{O}_{2}$, but also generate $\mathrm{H}_{2} \mathrm{O}_{2}$ (Passardi et al., 2004). Apoplastic peroxidases can either restrict or promote cell expansion (Tsukagoshi et al., 2010). In our study, one DEG encoding peroxidase (c33518_g1) was found to be differentially expressed in S6 relative to the $\mathrm{S} 7$ genotype at $24 \mathrm{~h}$.

Other DEGs that might be related to stress were also detected in our study of S6 and S7. These DEGs included genes encoding the two-component response regulator (c20693 g1) and cytochrome P450 (c31385_g1), serine/ threonine-protein kinase (c38177_g1 and

Genetics and Molecular Research 16 (1): gmr16019432 
c43920_g1), expansin (c49431_g1), DNA repair protein (c40074_g1), low-temperatureinduced protein (c41310_g1), $\mathrm{Ca}^{2+} / \mathrm{H}^{+}$exchange protein (c39501_g1), protein phosphatase $2 \mathrm{C}$ (c35944_g1), CBL-interacting protein kinase (c26640_g1 and c32118_g1), calcium-binding proteins (c31008_g1), and synaptobrevin, a member of the vesicle-associated membrane protein (VAMP) family(c40050_g1), etc.

Moreover, the functional classification of DEGs showed that out of 132 DEGs, approximately 30 unclassified and unknown-function genes were responsive to salinity stress. These 30 genes represented a larger set of genes, which might be the novel genes involved in salinity stress responses after the overexpression of $A h R a b G$ gene in peanut (Table S3). However, some genes with relatively lower transcript levels might not be detected using the RNA-seq analysis.

Collectively, our results indicate that the regulation of salinity stress resistance after overexpression of the $A h R a b G$ gene in peanut involves many aspects which are mentioned above. The information from this study can help us understand the mechanism of salinity resistance conferred by the expression of $R a b G$ gene in peanut.

\section{Conflicts of interest}

The authors declare no conflict of interest.

\section{ACKNOWLEDGMENTS}

Research supported by the National Natural Science Foundation of the China (Grant \#31301356, \#31571705, and \#31471542) and the Shandong Province Science and Technology Development Plan Project (Grant \# 2014GNC110002).

\section{REFERENCES}

Agarwal PK, Agarwal P, Jain P, Jha B, et al. (2008). Constitutive overexpression of a stress-inducible small GTP-binding protein PgRab7 from Pennisetum glaucum enhances abiotic stress tolerance in transgenic tobacco. Plant Cell Rep. 27: 105-115. http://dx.doi.org/10.1007/s00299-007-0446-0

Anders S and Huber W (2010). Differential expression analysis for sequence count data. Genome Biol. 11: R106 http:// dx.doi.org/10.1186/gb-2010-11-10-r106.

Battaglia M, Olvera-Carrillo Y, Garciarrubio A, Campos F, et al. (2008). The enigmatic LEA proteins and other hydrophilins. Plant Physiol. 148: 6-24. http://dx.doi.org/10.1104/pp.108.120725

Benjamini Y and Hochberg Y (1995). Controlling the False Discovery Rate: A Practical and Powerful Approach to Multiple Testing. J. R. Stat. Soc. Series B (Methodological) 57: 289-300.

Bogdanovic A, Bruckert F, Morio T and Satre M (2000). A syntaxin 7 homologue is present in Dictyostelium discoideum endosomes and controls their homotypic fusion. J. Biol. Chem. 275: 36691-36697. http://dx.doi.org/10.1074/jbc. $\underline{\mathrm{M} 006710200}$

Bolte S, Schiene K and Dietz KJ (2000). Characterization of a small GTP-binding protein of the rab 5 family in Mesembryanthemum crystallinum with increased level of expression during early salt stress. Plant Mol. Biol. 42: 923-936. http://dx.doi.org/10.1023/A:1006449715236

Brighouse A, Dacks JB and Field MC (2010). Rab protein evolution and the history of the eukaryotic endomembrane system. Cell. Mol. Life Sci. 67: 3449-3465. http://dx.doi.org/10.1007/s00018-010-0436-1

Fujita M, Fujita Y, Noutoshi Y, Takahashi F, et al. (2006). Crosstalk between abiotic and biotic stress responses: a current view from the points of convergence in the stress signaling networks. Curr. Opin. Plant Biol. 9: 436-442. http:// dx.doi.org/10.1016/j.pbi.2006.05.014

Gao J, Cheng H, Cai HB, An ZW, et al. (2010). In silico cloning and bioinformatics analysis of HbRD22 gene from Hevea brasiliensis. Chin. J. Trop. Crops 31: 1439-1445.

Genetics and Molecular Research 16 (1): gmr16019432 
Livak KJ and Schmittgen TD (2001). Analysis of relative gene expression data using real-time quantitative PCR and the 2(-Delta Delta C(T)) Method. Methods 25: 402-408. http://dx.doi.org/10.1006/meth.2001.1262

Lowy DR and Willumsen BM (1993). Function and regulation of ras. Annu. Rev. Biochem. 62: 851-891. http://dx.doi. org/10.1146/annurev.bi.62.070193.004223

Lu D, Wang T, Persson S, Mueller-Roeber B, et al. (2014). Transcriptional control of ROS homeostasis by KUODA1 regulates cell expansion during leaf development. Nat. Commun. 5: 3767. http://dx.doi.org/10.1038/ncomms4767

Mazel A, Leshem Y, Tiwari BS and Levine A (2004). Induction of salt and osmotic stress tolerance by overexpression of an intracellular vesicle trafficking protein AtRab7 (AtRabG3e). Plant Physiol. 134: 118-128. http://dx.doi. org/10.1104/pp.103.025379

Nahm MY, Kim SW, Yun D, Lee SY, et al. (2003). Molecular and biochemical analyses of OsRab7, a rice Rab7 homolog. Plant Cell Physiol. 44: 1341-1349. http://dx.doi.org/10.1093/pcp/pcg163

Nicolás C, Nicolás G and Rodríguez D (1998). Transcripts of a gene, encoding a small GTP-binding protein from Fagus sylvatica, are induced by ABA and accumulated in the embryonic axis of dormant seeds. Plant Mol. Biol. 36: 487491. http://dx.doi.org/10.1023/A:1005906601446

Nuoffer C and Balch WE (1994). GTPases: multifunctional molecular switches regulating vesicular traffic. Аnпи. Rev. Biochem. 63: 949-990. http://dx.doi.org/10.1146/annurev.bi.63.070194.004505

O'Mahony PJ and Oliver MJ (1999). Characterization of a desiccation-responsive small GTP-binding protein (Rab2) from the desiccation-tolerant grass Sporobolus stapfianus. Plant Mol. Biol. 39: 809-821. http://dx.doi. org/10.1023/A:1006183431854

Passardi F, Penel C and Dunand C (2004). Performing the paradoxical: how plant peroxidases modify the cell wall. Trends Plant Sci. 9: 534-540. http://dx.doi.org/10.1016/j.tplants.2004.09.002

Pereira-Leal JB and Seabra MC (2001). Evolution of the Rab family of small GTP-binding proteins. J. Mol. Biol. 313 : 889-901. http://dx.doi.org/10.1006/jmbi.2001.5072

Rajan N, Agarwal P, Patel K, Sanadhya P, et al. (2015). Molecular characterization and identification of target protein of an important vesicle trafficking gene AlRab7 from a salt excreting halophyte Aeluropus lagopoides. DNA Cell Biol. 34: 83-91. http://dx.doi.org/10.1089/dna.2014.2592

Sano H, Seo S, Orudgev E, Youssefian S, et al. (1994). Expression of the gene for a small GTP binding protein in transgenic tobacco elevates endogenous cytokinin levels, abnormally induces salicylic acid in response to wounding, and increases resistance to tobacco mosaic virus infection. Proc. Natl. Acad. Sci. USA 91: 10556-10560. http://dx.doi. org/10.1073/pnas.91.22.10556

Schimmöller F and Riezman H (1993). Involvement of Ypt7p, a small GTPase, in traffic from late endosome to the vacuole in yeast. J. Cell Sci. 106: 823-830.

Schmidt R, Schippers JH, Mieulet D, Obata T, et al. (2013). MULTIPASS, a rice R2R3-type MYB transcription factor, regulates adaptive growth by integrating multiple hormonal pathways. Plant J. 76: 258-273.

Song L, Li R, Xiang XH, Wang JS, et al. (2012). Over expression of stress-inducible small GTP-binding protein AhRab7 (AhRabG3f) in peanut (Arachis hypogaea L.) enhances abiotic stress tolerance. J. Food Agric. Environ. 10: 888-894.

Tsukagoshi H, Busch W and Benfey PN (2010). Transcriptional regulation of ROS controls transition from proliferation to differentiation in the root. Cell 143: 606-616. http://dx.doi.org/10.1016/j.cell.2010.10.020

Wichmann H, Hengst L and Gallwitz D (1992). Endocytosis in yeast: evidence for the involvement of a small GTPbinding protein (Ypt7p). Cell 71: 1131-1142. http://dx.doi.org/10.1016/S0092-8674(05)80062-5

Xing T, Higgins VJ and Blumwald E (1997). Race-specific elicitors of Cladosporium fulvum promote translocation of cytosolic components of NADPH oxidase to the plasma membrane of tomato cells. Plant Cell 9: 249-259. http:// dx.doi.org/10.1105/tpc.9.2.249

Zhang G, Chen M, Li L, Xu Z, et al. (2009). Overexpression of the soybean GmERF3 gene, an AP2/ERF type transcription factor for increased tolerances to salt, drought, and diseases in transgenic tobacco. J. Exp. Bot. 60: 3781-3796. http:// dx.doi.org/10.1093/jxb/erp214

Zhao YZ, Chen XL, Zeng DG, Yang CL, et al. (2015). Molecular cloning, characterization, and expression of Rab5B, Rab6A, and Rab7 from Litopenaeus vannamei (Penaeidae). Genet. Mol. Res. 14: 7740-7750. http://dx.doi. org/10.4238/2015.July.13.20

\section{Supplementary material}

Table S1. The summary of differential gene expression (DGE) library sequencing and analysis.

Table S2. Differentially expressed genes (DEGs) at five time points between S6 and S7 genotypes.

Genetics and Molecular Research 16 (1): gmr16019432 



vs $\mathrm{S} 7$.

Table S4. Primers for quantitative real-time PCR (qRT-PCR) analysis.

Table S5. Comparison of the expression levels of 8 differentially expressed genes (DEGs) as determined by RNAseq and real-time PCR analysis. 